\title{
Fraseología y traducción
}

\author{
Julia SeVilla MuÑOZ \\ UCM
}

Uno de los escollos que continuamente tiene que salvar el traductor, lo constituye la traducción de unidades fraseológicas, como los modismos, refranes, etc., pues poseen una estructura peculiar que los convierte en singulares; problema que se agrava por el reducido número de diccionarios bilingües y multilingües de fácil acceso dedicados a esta parcela lingǘstica.

Estas dificultades, sin embargo, no han de quitarnos la idea de aventurarnos en su estudio sistemático, ya que representan una parte importante del caudal de una lengua y recorren todos los tipos de discurso. El traductor, por tanto, ha de poseer la suficiente competencia linguística que le permita identificarlos en la lengua original, comprender su sentido y buscar su correspondencia en la lengua terminal.

EI análisis de los principales tipos de expresiones y enunciados fijos nos permitirá abordar los problemas traductológicos que plantean, así como intentar buscar técnicas y procedimientos para realizar la traducción de tales fenómenos lingüísticos.

Las dificultades que implica su traducción son de tres tipos:

- terminológicas y conceptuales;

- gramaticales y semánticas;

- lexicográficas y paremiográficas.

\section{PROBLEMA TERMINOLÓGICO Y CONCEPTUAL}

Existe una multitud de términos para denominar estas unidades lingüísticas: expresiones idiomáticas, expresiones fijas, modismos, idiotismos, locuciones, timos, muletillas, clichés, estereotipos, giros, frases hechas, dichos, refranes, proverbios, frases proverbiales,...; apelativos que hallamos en mayor o menor medida en otras lenguas, como el francés (expression idiomatique, expression figée, expression imagée, idiotisme, locution, cliché, tournure, phrase faite, phrase proverbiale, cli- 
ché, proverbe, dicton), el italiano (expressione idiomatica, modo di dire, idiotismo, locuzione, proverbio, modo proverbiali), el alemán (Phraseologismus, Redewen. dung, Redensart, Idiotismus, Sprichwort) o el inglés (Idiom, Proverb, Cliche).

Se suelen usar indistintamente tales designaciones por desconocimiento hacia esta parcela linguística o porque las expresiones y enunciados fijos presentan muchos rasgos comunes, lo que provoca una gran confusión, no sólo terminológica sino también conceptual: no sabemos cómo llamarlos y, menos aún, definirlos.

Todas estas configuraciones linguísticas contienen un elemento abstracto o imaginario y su significado final no resulta de la suma de los significados parciales de sus componentes; rasgo que Alberto Zuluaga (1980) llama la idiomaticidad, esto $\mathrm{es}$, el rasgo semántico propio de ciertas construcciones lingásticas fijas, cuyo sentido no puede establecerse a partir de los significados de sus elenentos componentes ni del de su combinación. La mayor o menor presencia de idiomaticidad nos permitirá establecer varios grados en el sentido de tales expresiones y enunciados. Así, el refrán En diciembre, leña y duerme, indica, con dos vocablos, lo que hacía el labriego en la época invernal. Su campo de aplicación es bastante reducido; no sucede lo mismo con el refrán Más vale pájaro en mano que ciento volando, cuyo mensaje puede aplicarse a un sinfín de situaciones más o menos próximas a su sentido literal relacionado con el mundo de la caza.

Además de la fijación y la idiomaticidad, estas expresiones y enunciados se caracterizan por su morfosintaxis peculiar - alejada a veces de las normas gramaticales--, por estar memorizadas en competencia y ser propias de una lengua. En algunos casos, se produce una similitud de significante entre lenguas de un mismo tronco, como el español y el francés: A la vulpeja dormida, no le cae nada en la boca, A renard endormi, rien ne lai tombe dans la gueule. Ser blanco como la nieve / Ser más blanco que la nieve, Etre blanc comme neige. Sin embargo, no son tantos como pudiera parecer a simple vista. Así, si bien la idea de imposibilidad se expresa, tanto en español como en francés, a través del mismo animal, la acción verbal no coincide: Cuando meen las gallinas, Quand les poules auront des dents. La distancia formal aumenta, si pensamos que, en español, se utiliza más la frase Cuando las ranas crien pelo.

El uso, precisamente, establece una primera distinción entre las expresiones y los enunciados fijos, pues, es evidente la caída en desuso de éstos últimos, en particular, los refranes, los cuales están sufriendo alteraciones formales (generalmente por fmes cómicos; por ejemplo: En abril, billetes de mil. Ojos que no ven, gabardina que te roban) o están siendo sustituidos por otras unidades, como el eslogan. Por otra parte, las expresiones son combinaciones idiomáticas, carentes de tono moralizante y estables de dos o más palabras que constituyen elementos oracionales del discurso:

- Locución adjetiva (adjetivo): de tomo y lomo, de pacotilla.

- Loc. adverbial (adverbio): de repente, a tontas y a locas.

- Loc conjuntiva (conjunción): por consiguiente, con tal que, a pesar de que.

- Loc. interjectiva (interjección): jay de mí! iválgame Dios!

- Loc. prepositiva (preposición): en tomo a, en pos de. 
Los enunciados fijos, en cambio, destacan por la brevedad, el mensaje sentencioso y estar engastados en el discurso.

Pese a estas diferencias, cabe señalar que, a veces, las fronteras no resultan tan nítidas, porque algunas expresiones parecen haberse desgajado de este tipo de enunciados o constituyen realizaciones más libres de éstos. La expresión $I r / v e n i r$ por lana y salir trasquilado constituye una locución verbal doble que conserva todavía la lección moral del refrán El camero encantado, que fue por lana y volvió trasquilado. Del mismo modo, Mañana será otro día, considerado por algunos un modismo (Sánchez Anaya, 1988), aconseja tener paciencia y optimismo, igual que el enunciado Mañana será otro día, y verá el tuerto los espárragos.

No resulta fácil establecer límites inequívocos entre ellos; mas dicho problema puede solucionarse, a nuestro juicio, mediante la búsqueda de un archilexema que nos permita levantar barreras conceptuales entre ellos, clasificarlos y definirlos. Nuestras investigaciones nos han llevado a usar dos vocablos con carácter genérico para aludir a la expresiones y enunciados fijos: las expresiones idiomáticas y las paremias, respectivamente.

Los rasgos de los archilexemas expresión idiomática y paremia nos permitirán arrojan un poco de luz desde el punto de vista conceptual y terminológico con la localización y definición de los principales tipos, así como su correspondencia en otras lenguas; aspectos que hemos abordado en diversos trabajos y que siguen siendo objeto de nuestro estudio.

\section{Problema MORFOSINTÁCTICO Y SEMÁNTICO}

Dificulta también la traducción de las expresiones y enunciados fijos su estructura gramatical peculiar, puesto que se alejan muchas veces de las normas linguíslicas, cuentan con la presencia de arcaísmos o sufren -en el caso de las paremias populares - alteraciones vocálicas con fines rítmicos: El mur que no sabe más que un horado, presto lo toma el gato; Lo que has de dar al rato, dáselo al gato, e, incluso, modificaciones gramaticales y estructurales, como se aprecia en estos refranes utilizados por Sancho Panza y Don Quijote:

[...] ¡Esas burlas, a un cuñado; que soy perro viejo, y no hay conmigo tus, tus! (IJ, 69, 1132).

- Por esa trova - dijo Sancho- no se puede saber nada, si ya no es que por ese hilo que está ahí se saque el ovillo de todo $(\mathrm{I}, 23,300)$.

[Don Quijote]... escuchémosle, que por el hilo sacaremos el ovillo de sus pensamientos, si es que canta; que de la abundancia del corazón habla la lengua $(11,12,710)$.

En el primer refrán (A perro viejo, no hay tus, tus) se ha producido una alteración morfosintáctica; en el segundo (Por el hilo se saca el ovillo), la adjunción de elementos explicativos conforme a la situación y el cambio de la aplicación de las personas gramaticales. 
Tales modificaciones obstaculizan enormemente la localización de las paremias; problema que se agrava si pensamos en que, incluso en su lengua materna, el traductor no siempre es capaz de comprender del todo el mensaje de unos enunciados que pueden poseer varios sentidos y responder a distintas ideas clave, según el contexto y la situación. Además, en la actualidad, es reducido el número de usuarios de los refranes y otros enunciados sentenciosos populares y cada vez resulta más dif́cil recurrir a la fuente oral para consultarle acerca del sentido y la función de la paremia en cuestión. No sucede lo mismo con las expresiones idiomáticas, ya que, por lo general, el traductor las reconoce y comprende sin dificultad.

\section{PROBLEMA LEXICOGRÁFICO Y PAREMIOGRÁFICO}

Para hallar la traducción de tales fenómenos linguiísticos se suele recurrir al diccionario, pero este camino no siempre nos lleva a una solución y, mucho menos, a una solución fiable.

Las diferencias mencionadas entre las expresiones idiomáticas y las paremias, especialmente los refranes, se reflejan en las obras que las registran. Los diccionarios de lengua bilingüe son ricos en expresiones idiomáticas, pero muy pobres en paremias populares; en cambio, la abundancia en repertorios paremiográficos multilingües contrasta con la escasez en diccionarios plurilingües de expresiones idiomáticas. En cuanto a las obras bilingües especializadas, éstas escasean en ambos campos. En efecto, pocos son los repertorios bilinguies o multilingües dedicados exclusivamente a las expresiones idiomáticas; los que existen suelen agruparlas por palabras clave y las acompañan del significado, sinónimos, un ejemplo y la correspondencia en la o las otras lenguas, como observamos en el repertorio de Mariano S. Anaya (1988):

PIES: Poner los pies en polvorosa. Pies para qué os quiero. Tomar las de Villadiego. Salir pitando.

Salir huyendo a toda prisa.

Cuando Montoya supo que la policía le estaba buscando, puso los pies en polvorosa.

F. Prendre la poudre d'escampette. Faire le mur. Décamper. Prendre la clé des champs. Avoir le feu au derrière. Vider les lieux. Lever le pied. Pendre ses jambes à son cou. Démenager à la cloche de bois.

I. To take off. To run off. To beat it. To get away quick. To get out quick. To turn heels and run.

Sin embargo, no se incluyen indicaciones acerca de su frecuencia, el nivel de lengua, el registro al que pertenecen, ni tampoco del uso; datos, a nuestro juicio, indispensables para lograr una buena traducción. Así, muchas expresiones aluden a la idea de huir precipitadamente (Salir echando leches, Salir a toda pastilla, Salir pitando, Echar patas,...), mas no todas pueden usarse indistintamente porque pertenecen a diferentes registros y niveles de lengua y, además, ha de tenerse en cuenta si 
se trata de una forma en uso o en desuso. Se ha de buscar obviamente una correspondencia no sólo de significado, sino también de registro, de nivel de lengua y de uso. La falta de atención a estos datos puede llevarnos a una traducción errónea.

La escasez de obras de este tipo se debe a lo laborioso de su elaboración y a que los diccionarios de lengua bilingües registran la traducción — bastante acertada, por lo general - de las expresiones idiomáticas de uso corriente; pero, por la naturaleza de estos diccionarios, no aparecen los datos mencionados que consideramos importantes para el proceso de traducción de estas expresiones. Otro problema, lo constituye la dificultad que supone presentar un corpus actualizado de estas expresiones, pues se renuevan con facilidad y conviven con muchos sinónimos de registros distintos.

En cuanto a las paremias, los diccionarios bilingües de lengua publicados en las últimas décadas son muy pobres en traducción de paremias. Así, a partir de la edición de 1987, Ramón García-Pelayo y Jean Testas dedican las páginas centrales del Dictionnaire Français-Espagnol (Paris: Larousse) a repertoriar locuciones, refranes y frases proverbiales con su correspondencia francesa. Sólo hay registradas unas 300 paremias, una cantidad pequeña si tomamos como punto de referencia los 65.000 refranes castellanos recopilados por Luis Martínez Kleiser en su Refranero General Ideológico Español (Madrid, 1953). El traductor trata entonces de recurrir a las colecciones paremiográficas bilingües y multilingües, pero éstas presentan bastantes deficiencias, como la ausencia de significado.

No hay muchas colecciones que tomen como punto de partida las paremias españolas para buscar su correspondencia en otra lengua. De 1907 y 1911, datan, por ejemplo, los dos primeros tomos del Doctrinal de Juan del Pueblo, en el que Fermín Sacristán agrupa refranes por materias ordenadas alfabéticamente y acompañados de su correspondencia en los dialectos y lenguas peninsulares españolas, así como en latín y en las lenguas romances; pero, desgraciadamente sólo llegó a publicar hasta la letra $c$. Mucho más reciente en el tiempo y de fácil acceso, es la confeccionada por Jorge Sintes Pros: Diccionario de aforismos, proverbios y refranes, con la interpretación para su empleo correcto, y con la equivalencia en siete idiomas: Portugués, Francés, Italiano, Inglés, Alemán, Latín y Catalán (1954). Sin embargo, el título no responde a la realidad, ya que, de las 4.600 entradas paremiológicas, sólo 1.600 van acompañadas por su correspondencia; de las 3.000 restantes, sólo se da el significado. Se observa la ausencia de paremias españolas muy conocidas y la repetición de entradas paremiológicas provocadas por existir dos criterios de clasificación: alfabético por idea o por palabra clave. Así pues, no disponemos de una obra española actualizada y lo más completa posible que cubra este campo lingüístico.

En lo que atañe a las colecciones paremiográficas plurilingües publicadas en otros países, en las que aparece el español, contiene una serie de inconvenientes que dificultan o desaconsejan su consulta:

1) Conocen una escasa difusión y, para localizarlas, hay que dirigirse a bibliotecas especializadas o a la Biblioteca Nacional, como sucede con el reperto- 
rio de Y. P. de Dony, Léxico del lenguaje figurado en cuatro idiomas (castellano, français, english, deutsch) (1951). Todo ello supone una gran pérdida de tiempo, pues, en la mayoría de los casos, sólo se pretende buscar la correspondencia de una sola paremia.

2) Normalmente, los fondos de las bibliotecas españolas no son ricos en obras de este tipo y, al ser diccionarios, están excluidos de préstamo y, por tanto, del préstamo interbibliotecario.

3) Dado que son plurilingües, cuanto más lenguas abarcan menor es el número de paremias registradas y menor el número de probabilidades de encontrar la paremia cuya correspondencia en otra lengua se pretende hallar. Constituyen obras cerradas (v.gr.: los 1.483 proverbios y expresiones proverbiales italianas recogidos alfabéticamente por Augusto Arthaber en el Dizionario comparato di proverbie modi proverbiali, 1981, acompañados, siempre que le ha sido posible, de la correspondencia en latín, francés, español, alemán, inglés y griego antigưo).

4) La correspondencia consiste, en la mayoría de los casos, en una traducción palabra por palabra de la paremia que sirve de punto de partida, procedimiento seguido, por ejemplo, por Gérard Ilg (Proverbes français suivis des équivalents en allemand, anglais, espagnol, italien, néerlandais, 1960 y por Jerzy Gluski (Proverbs. A Comparative Book of English, French, German, Spanish and Russian Proverbs with a Latin Appendix, 1971).

5) Muy pocas obras de esta índole acompañan las paremias de una explicación acerca de su significado.

De los escasos repertorios bilinguies paremiográficos, cabe destacar la magnífica, pero poco difundida, Selección de refranes y sentencias elaborada por Jesús Cantera y Eugenio de Vicente (1983-84). 4.346 paremias españolas y francesas dispuestas por orden alfabético y en dos tomos (francés-español y español-francés).

$\mathrm{El}$ traductor no dispone, por tanto, de un repertorio paremiográfico plurilingüe completo, fiable y de fácil acceso para realizar una consulta puntual.

\section{CONCLUSIONES}

La inadecuada traducción, la incompleta información o la inaccesibilidad de muchos de los diccionarios especializados bilingües y multilingües, llevará al traductor, en no pocas ocasiones, a tener que resolver por sí solo el problema terminológico. Para salir airoso de esta empresa, deberá poseer la suficiente competencia fraseológica (paremiológica y de las expresiones en desuso), tanto en la lengua extranjera como en su lengua materna, con el fin de localizarlas, adquirir el grado de comprensión necesario para saber expresar en su lengua esa riqueza léxica y reflejar las posibles modificaciones producidas por fines lúdicos o cómicos. La adquisición de esta competencia no supone una empresa fácil, pues, en el caso de las expresiones idiomáticas se ha de tener en cuenta el uso, el registro y el nivel de lengua, y, en cuanto a las paremias, no se ha de olvidar las alteraciones formales y los 
cambios de sentido que pueden sufrir en función de la situación y del contexto en que aparecen.

La búsqueda de una traducción válida de estos fenómenos lingüísticos, ha de basarse en los datos aportados por los diccionarios monolingües y en la fuente oral; datos que se plasmarán en fichas creadas ad hoc.

El modelo de ficha para la sistematización de las expresiones idiomáticas, tomando como punto de partida el español, es la siguiente:

Texto: Poner pies en polvorosa

Significado: Huir precipitadamente

Ejemplo: Cuando Pérez supo que el inspector de Hacienda había preguntado por él puso pies en polvorosa.

Registro: Coloquial

Uso: Poco usado

Sinónimos: Echar patas. ¡Pies para qué os quiero! Poner tierra por medio. Salir a toda pastilla. Salir echando leches. Salir echando chispas. Salir por pies. Salir pitando. Salir como un cohete. Coger/tomar las de Villadiego. Salir como alma que lleva el diablo.

Antónimos: Aguantar el tipo. Aguantar el tirón. Quedarse pasma(d)o. Quedarse de piedra. Seguir en la brecha. Seguir al pie del cañón.

Correspondencia: Prendre la poudre d'escampette. Prendre la clé des champs.

Fuente: oral (personas mayores)

El modelo de ficha que proponemos para las paremias con el francés, por ejemplo, como lengua de partida, es éste:

Texto: Le singe, fât-il vêtu de pourpre, est toujours singe

Variantes formales: Un singe vêtu de pourpre est toujours un singe.

Idea clave: Apariencias

Significado: No todo es lo que parece.

Sinónimos: Le singe est toujours singe, et füt-il dégaisé en prince.

Serpent qui change de peau est toujours serpent.

Lavez chien, peignez chien, - Toutefois n'est chien que chien.

Antónimos: Parez un buisson, il ressemblera un baron.

Correspondencia:

- literal: Aunque la mona se vista de seda, mona se queda.

- conceptual: La mona es siempre mona, aunque la vistan de real persona.

- antónimo - Compón el sapillo, parecerá bonillo.

Fuente: escrita y oral.

No resulta fácil dar ejemplos de las paremias engastadas en el discurso, pues el usuario puede alterarlas y manipularlas por fines lúdicos o irónicos, con lo que pueden producirse cambios de sentido. 
En lo que respecta a los sinónimos y antónimos, sólo hemos registrado los referentes a animales; pero, existen muchos más. Por otra parte, dado que esta ficha es un modelo, no hemos especificado las fuentes escritas, las cuales aparecerían en abreviaturas. Además, estas fichas, pertenecientes a colecciones bilingües, pueden modificarse según el campo considerado prioritario. Así, la ficha modelo para un repertorio multilingüe podría ser:

Aunque la mona se vista de seda, mona se queda.

Apariencias. «No todo es lo que parece».

* La mona es siempre mona, aunque la vistan de real persona.

\# Compón el sapillo, parecerá bonillo.

Fuentes: escrita y oral.

fr. Le singe, fût-il vêtu de pourpre, est toujours singe.

it. La scimmia è sempre scimmia, anco vestita di seta.

It. Simia semper simia, / Etsi aurea gestet insignia.

in. An ape's an ape, a varlet's a varlet. / Though they be clad in silk or scarlet.

al. Affen bleiben Affen, wenn man sie auch in Sammet kleidet.

wolof. Bant, lu mu yágg ci dex, du tax mua soppiku jasig.

[* El tronco de un árbol, aunque dure en el agua, nunca se convierte en cocodrilo]

La ficha para repertorios multilingües de expresiones idiomáticas también puede variar. He aquí una posibilidad para la sistematización de las comparaciones, un grupo muy numeroso dentro de las expresiones idiomáticas:

\begin{tabular}{|c|c|c|}
\hline $\begin{array}{l}\text { COMPARACIÓN } \\
\text { SER MÁS BLANCO QUE... } \\
\text { (Personas) }\end{array}$ & $\begin{array}{l}\text { BLANCO } \\
\text { Aspecto } \\
\text { Color de la pie: }\end{array}$ & $\begin{array}{l}\text { COLOR } \\
\text { Enfermedad - Miedo }\end{array}$ \\
\hline \multicolumn{2}{|l|}{$\begin{array}{l}\text { ESPAÑOL: Ser más blanco que } \\
\text { la pared, el papel }\end{array}$} & la nieve, la leche \\
\hline & $\begin{array}{l}\text { la azucena } \\
\text { un calamar } \\
\text { el pecho de una aspirina } \\
\text { el pan candeal }\end{array}$ & un muerto, un cadáver \\
\hline FRANCÉS: Blanc comme & $\begin{array}{l}\text { neige } \\
\text { un calchet d'aspirine } \\
\text { un lys } \\
\text { le coton (Côte-d'Ivoire) }\end{array}$ & $\begin{array}{l}\text { un linge, un drap } \\
\text { un cadavre, un/la mort }\end{array}$ \\
\hline PORTUGUÉS: Branco como & a neve & un lençol \\
\hline ITALIANO: Bianco come & la neve, il latte & un cencio Javato \\
\hline INGLÉS: & As white as snow & a ghost, death \\
\hline ALEMÁN: & Weiß wie Schnee & Leiche \\
\hline
\end{tabular}


Esta ficha es susceptible de completarse con más lenguas, más sinónimos, una columna dedicada a las formas aplicadas a los objetos, concretamente al aspecto, al grado de limpieza. Por ejemplo: Ser/estar más blanco que la cal.

En trabajos anteriores, hemos desarrollado los criterios que nos han llevado a elegir los campos que componen estas fichas, que tratan de proporcionar un instrumento para facilitar la traducción de expresiones idiomáticas y paremias, un método de sistematización que admite mejoras y, lo más importante, que abre nuevas vías de investigación.

\section{REFERENCIAS BIBLIOGRÁFICAS}

ArTHABER, A. (1981): Dizionario comparato di proverbi e modi proverbiali. Milán: Hoepli. BLuM, G. (1989) Les Idiomatics (français-espagnol), dessins de Nestor Salas. Paris: Éditions du Seuil.

CANTERA, J. \& DE VICENTE, E. (1983-84): Selección de refranes y sentencias. Madrid: Editorial Complutense.

Cervantes SaAvedra, M. de (ed. de 1994): Don Quijote de la Mancha. Ed. de Martín de Riquer. Barcelona: RBA Editores.

DONY, Y. P. de (1951): Léxico del lenguaje figurado en cuatro idiomas (castellano, français, eng(ish, deutsch). Buenos Aires: Desclée de Brouwer.

Ferrer A.s, A.; Barrios, A. (1948): Mil locuciones españolas. Paris.

Gluski, J. (1971): Proverbs. A Comparative Book of English, French, German, Spanish and Russian Proverbs with a Latin Appendix. Amsterdam: Elservier Publishing Compagny.

ILG, G. (1960): Proverbes français waivis des équivalents en allemand, anglais, espagnol, italien. néerlandais. Amsterdam-London-New York-Princeton: Elservier Publishing Compagny.

LAFLEUk, B. (1984): Dictionnaire des Expressions. Paris: Bordas,

LoZANO, C. W. (1992): «Aproximación al problema de las expresiones idiomáticas y su trađucción», Sendebar, 3: 141-156.

— (1993): «La traducción de las expresiones idiomáticas: francés-español».

MakTinez Klesser, L. ([1953) ed. 1978): Refranero General Ideológico Español. Madrid: Editorial Hernando.

MoI INER, M. (1982): Diccionario de uso del español. Madrid: Gredos.

SánCiez Anaya, M. (en colaboración con Ch. Guyomard y H. ANderson) (1988): $\mathrm{Mil}$ modismos y origen de muchos de ellos. Salamanca: Mariano Sánchez Anaya.

Sevilla Muño7, J. (1987-88): «Los universales paremiológicos en dos paremias heroicas» (una francesa y otra española), Revista de Filologia Románica, v: 221-234.

- (1988): Hacia una aproximación conceptual de las paremias francesas y españolas. Madrid: Ed. Complutense.

- (1990): «La traducción al español de algunas parcmias francesas», H Encuentros Complatenses en tomo a la traducción [Madrid, 1988|, pp. 145-150. Madrid: Editorial de la Universidad Complutense.

- (1991): «Propuesta de sistematización paremiográfica», Revista de Filologia Románica, 8: 31-39.

- (1992): «Propuesta de sistematización de los dictons», Revista de Filología Francesa. 1 : 175-187. 
-.. (1992): «Algunas referencias sobre las traducciones paremiológicas entre el francés y el español», Livius. Revista de Estudios de Traducción (Univ. de León), 2, pp. 95-105.

- (1992): «La terminologie parémiologique française et sa correspondance espagnole», Terminologie et Traduction, publiée par la Commission de la Communauté Européenne [Colloque International «Phraséologie et Terminologie» en traduction et en Interprétafion, Ginebra, octubre de 1991$\} \mathbf{n}^{\circ}$ 2/3: 331-343.

- (1992); «Fuentes paremiológicas francesas y españolas en el siglo XVI», Rev. de Fil. Románica, 9: 103-123.

- (1993): «La noción de 'expresión idiomática' en francés y en español» [en col. con Alvaro Arroyo], Revista de Filología Francesa, 4: 247-261.

- (1993): «Las paremias españolas: clasificación, definición y correspondencia francesa», Paremia, n. 2 [Actas del Coloquio Internacional aOralidad y escritura: literatura paremiologica y refranero", Orléans, 19-20 de noviembre de 1993!, Paremia, 2: 15-20.

- (1993): *Fuentes paremiológicas francesas y españolas en la primera mitad del siglo XVlI», Rev. de Fil. Románica, 10: 361-373.

- (1994)): «Fuentes paremiológicas en la segunda mitad del siglo XVIJ», Rev. de Fil. Románica, 11-12: 433-442.

- (1994): «Fuentes paremiológicas lrancesas y españolas en el siglo XVIII», Rev. de Fil. Francesa, 5: 299-311.

- (1995): «Didáctica de la traducción al español de paremias francesas» [en col. con Teresa Quevedo Aparicio], Didáctica (Lengua y Literatara), 7: 133-148.

- (1994): «La traducción en las colecciones paremiográlicas plurilingües» /en col. con Mcrcedes Burrel Arguis], Livius. Revista de Estudios de Traducción, 5: 189-198.

- (1994-95): «La traducción y la didáctica de las expresiones idiomálicas (francés-español)» [en col. con Antonio González Rodríguez], Equivalences (Revista del Institut Supérieur de Traducteurs et Interprètes de Bruselas), vol. 24/2 y 25/1-2: 171-182.

- (1997): «La competencia paremiológica: los refranes» (en col. con Juan Carlos Díaz), Proverbiam, 14.

Sintes Pros, J. ([1954] 1982): Diccionario de aforismos, proverbios y refranes, con la interpretación para su empleo correcto, y con la equivalencia en siete idiomas: Portugués, francés, Italiano, Inglés. Alemán, Latín y Catalán. Barcelona: Editorial Sintes.

ZuLUAGA, A. (1980): Introducción al estudio de las expresiones fijas, cap. IV, pp. 121-135. Frankfurt a. M./Bern: Peter D. Lang. 